\title{
RMI, a new OB-fold complex essential for Bloom syndrome protein to maintain genome stability
}

\author{
Dongyi Xu, ${ }^{1,6}$ Rong Guo, ${ }^{1,6}$ Alexandra Sobeck, ${ }^{2}$ Csanad Z. Bachrati, ${ }^{3}$ Jay Yang, ${ }^{4}$ Takemi Enomoto, ${ }^{5}$ \\ Grant W. Brown, ${ }^{4}$ Maureen E. Hoatlin, ${ }^{2}$ Ian D. Hickson, ${ }^{3}$ and Weidong Wang ${ }^{1,7}$ \\ ${ }^{1}$ Laboratory of Genetics, National Institute on Aging, National Institutes of Health, NIH Biomedical Research Center, \\ Baltimore, Maryland 21224, USA; ${ }^{2}$ Department of Biochemistry and Molecular Biology, Oregon Health and Science \\ University, Portland, Oregon 97239, USA; ${ }^{3}$ Weatherall Institute of Molecular Medicine, University of Oxford, John Radcliffe \\ Hospital, Oxford OX3 9DS, United Kingdom; ${ }^{4}$ Department of Biochemistry, University of Toronto, Toronto, Ontario M5S \\ 1A8, Canada; ${ }^{5}$ Molecular Cell Biology Laboratory, Graduate School of Pharmaceutical Sciences, Tohoku University, \\ Sendai 980-8578, Japan
}

\begin{abstract}
BLM, the helicase mutated in Bloom syndrome, associates with topoisomerase $3 \alpha$, RMI1 (RecQ-mediated genome instability), and RPA, to form a complex essential for the maintenance of genome stability. Here we report a novel component of the BLM complex, RMI2, which interacts with RMI1 through two oligonucleotide-binding (OB)-fold domains similar to those in RPA. The resulting complex, named RMI, differs from RPA in that it lacks obvious DNA-binding activity. Nevertheless, RMI stimulates the dissolution of a homologous recombination intermediate in vitro and is essential for the stability, localization, and function of the BLM complex in vivo. Notably, inactivation of RMI2 in chicken DT40 cells results in an increased level of sister chromatid exchange (SCE) - the hallmark feature of Bloom syndrome cells. Epistasis analysis revealed that RMI2 and BLM suppress SCE within the same pathway. A point mutation in the OB domain of RMI2 disrupts the association between BLM and the rest of the complex, and abrogates the ability of RMI2 to suppress elevated SCE. Our data suggest that multi-OB-fold complexes mediate two modes of BLM action: via RPA-mediated protein-DNA interaction, and via RMI-mediated protein-protein interactions.
\end{abstract}

[Keywords: Bloom syndrome; BLM; BLAP75; RMI1; RMI2; Topoisomerase 3 $\alpha$ ]

Supplemental material is available at http://www.genesdev.org.

Received June 24, 2008; revised version accepted August 19, 2008.

Bloom syndrome (BS) is a rare autosomal genetic disease characterized by growth retardation, reduced fertility, immunodeficiency, and predisposition to the development of various types of cancer (German 1993; Bachrati and Hickson 2008). Cells from BS patients display genomic instability characterized by an elevated frequency of sister chromatid exchanges (SCEs). The gene mutated in this disease, $B L M$, encodes a member of the RecQ helicase family that includes WRN and RECQL4, mutations of which also cause genomic instability and cancer predisposition in Werner syndrome and Rothmund-Thomson syndrome, respectively. The importance of this protein family in defending genome integrity is further supported by studies in other eukaryotic species, including yeast and Drosophila (Bachrati and Hickson 2008).

The biochemical activities of BLM suggest that it

\footnotetext{
${ }^{6}$ These authors contributed equally to this work.

${ }^{7}$ Corresponding author.

E-MAIL wangw@grc.nia.nih.gov; FAX (410) 558-8331.

Article is online at http://www.genesdev.org/cgi/doi/10.1101/gad.1708608.
}

plays an important role in homologous recombination (HR)-dependent DNA repair (Wu and Hickson 2006). First, BLM is capable of migrating DNA structures that resemble intermediates generated during $\mathrm{HR}$, such as model Holliday junctions (HJs) and D-loops (Karow et al. 2000; van Brabant et al. 2000; Bachrati et al. 2006). Second, BLM can disrupt the RAD51-coated presynaptic filament and promote DNA repair synthesis by DNA polymerase $\eta$ (Bugreev et al. 2007). Third, BLM can convert a stalled replication fork into an $\mathrm{HJ}$ through fork regression, which may facilitate the restart of blocked forks (Ralf et al. 2006). Fourth, BLM and its partner, topoisomerase $3 \alpha$ (Topo $3 \alpha$ ), cooperate to "dissolve" a double $\mathrm{HJ}(\mathrm{dHJ})$ in a manner that suppresses crossovers (Wu and Hickson 2003; Plank et al. 2006). BLM is thereby instrumental in preventing aberrant recombination, elevated SCEs, and genome rearrangements, which occur in BLM-deficient cells.

We previously purified BLM from human HeLa cells, and showed that it is present in at least three multiprotein complexes (Meetei et al. 2003). Eight Fanconi anemia proteins, Replication Protein A (RPA), and MLH1 are 
Xu et al.

components of some of these complexes (Meetei et al. 2003, 2005), but two proteins are present in all BLMassociated complexes: Topo $3 \alpha$, and a protein first named as BLAP75 (Yin et al. 2005), but later renamed RMI1 (RecQ-mediated genome instability) (Wu et al. 2006). RMI1 has been noted to contain an oligonucleotide-binding (OB)-fold domain (Yin et al. 2005), and can strongly stimulate dissolution of $\mathrm{dHJ}$ by BLM and Topo $3 \alpha$ (Raynard et al. 2006; Wu et al. 2006).

Evidence from both human and yeast studies suggests that BLM (or its ortholog Sgs1), Topo $3 \alpha$, and RMI1 form an evolutionarily conserved complex that works coordinately to process a diverse array of DNA structures in eukaryotes (Chang et al. 2005; Mullen et al. 2005; Yin et al. 2005; Raynard et al. 2006, 2008; Wu et al. 2006; Bussen et al. 2007; Chen and Brill 2007). This trimeric complex has been referred to as BTB (BLM-Topo $3 \alpha-B L A P 75)$ or RTR (RecQ-Topo $3 \alpha-\mathrm{RMI} 1$ ) in previous studies. We will use the term BLM complex to refer to the tetrameric complex (BLM-Topo 3 $\alpha$-RMI1-RMI2), which includes a novel component, RMI2. RMI2 and RMI1 form a stable subcomplex, named RMI, through interactions between a pair of OB-fold domains that resemble those in RPA. Interestingly, RMI differs from previously described multi-OB-fold complexes in that it lacks detectable ssDNA-binding activity. Instead, it mediates critical protein-protein interactions for the BLM complex. Our data suggest that multi-OB-fold complexes participate in two modes of actions in the BLM complex: protein-DNA interaction via RPA, and protein-protein interactions via RMI.

\section{Results}

\section{RMI2 is an integral component of the BLM complex}

All BLM-associated complexes contain Topo $3 \alpha$ and RMI1 (Meetei et al. 2003; Yin et al. 2005), and silverstaining analyses revealed the presence of an additional $20-\mathrm{kDa}$ protein in the complexes immunoisolated using either a BLM or an RMI1 antibody (see Fig. 1A in Yin et al. 2005). This protein was previously overlooked because its gel mobility is similar to that of immunoglobulin light chain. We repeated the immunoprecipitation with the same two antibodies, and found that the level of the $20-\mathrm{kDa}$ protein was in approximately stoichiometric amounts with other BLM complex components (Fig. 1A). Mass spectrometry identified this polypeptide as LOC116028, a hypothetical protein with no known function (gene name: C16orf75; accession no. NP_689521). We named this protein RMI2, because it directly interacts with RMI1 to form a multi-OB-fold complex (see below). An antibody against LOC116028 recognized this polypeptide in complexes immunoprecipitated by either BLM or RMI1 antibodies, suggesting that LOC116028 is RMI2 (Fig. 1B).

We infer that RMI2 is an integral component of the BLM complex based on the following evidence. First, RMI2 was coimmunoprecipitated by antibodies against
A
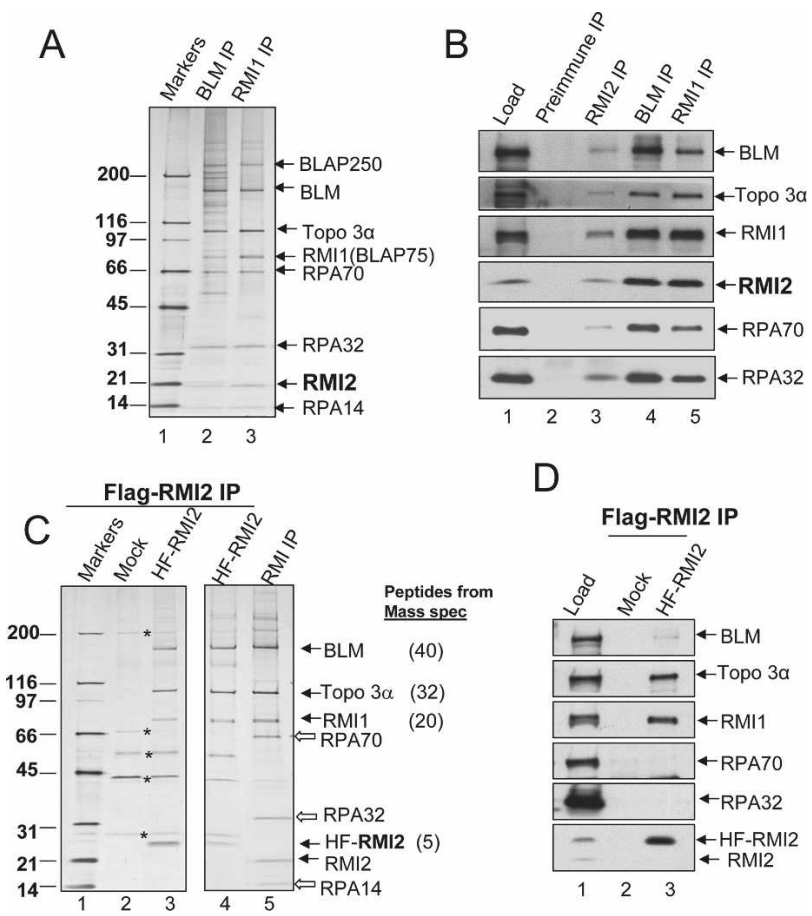

D

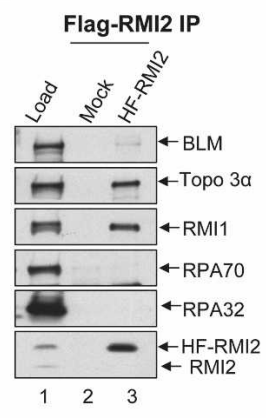

Figure 1. RMI2 is an integral component of BLM complexes. (A) A silver-stained SDS gel showing the presence of RMI2 in complexes immunoprecipitated by both BLM and RMI1 antibodies. The identity of different BLM components has been determined previously by mass spectrometry and immunoblotting (also see $B$ ). (B) Immunoblotting to show that RMI2 coimmunoprecipitates with other components of BLM complexes. Immunoprecipitation (IP) with preimmune serum was included as a negative control. The nuclear extract input (Load) is also shown. $(C, D)$ A silver-stained SDS gel $(C)$ and immunoblotting $(D)$ show that 6-histidine and Flag double-tagged RMI2 (HFRMI2) coimmunoprecipitated with BLM, Topo $3 \alpha$, and RMI1, but not RPA. The major polypeptides corresponding to the BLM components identified by mass spectrometry, and the numbers of peptides identified are indicated on the right of the figure. As a control, mock IP (Mock) was performed by using regular HeLa cells that do not express HF-RMI2. Several contaminating polypeptides are marked by asterisks. IP was performed using Flag antibody. We failed to detect RPA in the BLM complex isolated using the HF-RMI2 antibody $(C, D)$, even though RPA was detectable in the complex immunoprecipitated using the antibody against endogenous RMI2 $(B)$. One possibility is that the existence of the HF tag interferes with the interaction between HF-RMI2 and RPA.

multiple components of the BLM complex, including BLM, RMI1, RPA32, and RPA70 (Fig. 1A,B; data not shown). Second, a reciprocal immunoprecipitation with an RMI2 antibody coprecipitated the BLM complex proteins, including BLM, RMI1, Topo $3 \alpha$, and RPA (Fig. 1B). Third, we established a HeLa cell line stably expressing RMI2 that was double-tagged with a 6-histidine and a Flag epitope (termed HF-RMI2); and the major polypeptides coimmunoprecipitated with HF-RMI2 were identified as BLM, RMI1, Topo $3 \alpha$, and RMI2 by both mass spectrometry and immunoblotting (Fig. 1C,D). 
Fractionation of nuclear extract by gel filtration chromatography and subsequent immunoprecipitation further revealed that RMI2 is present in two complexes: One consists of BLM, Topo $3 \alpha$, RMI1, RMI2, RPA, and BLAP250, whereas the other one contains only RMI1, RMI2, and Topo $3 \alpha$ (Supplemental Fig. 1). The fact that the two RMI proteins can form an independent complex with Topo $3 \alpha$ implies that they may facilitate Topo $3 \alpha$ function, which is consistent with previous findings that RMI1 promotes the DNA-binding and catalytic activity of Topo $3 \alpha$ (Wu et al. 2006; Chen and Brill 2007).

\section{RMI2 and RMI1 interact through a pair of $O B$ domains that resemble those in RPA}

RMI2 contains an OB-fold nucleic acid-binding domain, termed RMI2-OB3 (the names OB1 and OB2 are used here for the domains in RMI1) (Fig. 2A). While RMI1 is present in the majority of eukaryotes, RMI2 is only present in vertebrates and plants, but absent in invertebrates and yeast (Fig. 2B), hinting that RMI2 is needed in higher eukaryotes that have more complex genomes.

We found that RMI2-OB3 is most similar to the OB domain " $\mathrm{D}$ " in RPA2 (Fig. 2A,C). RPA2 is one of the
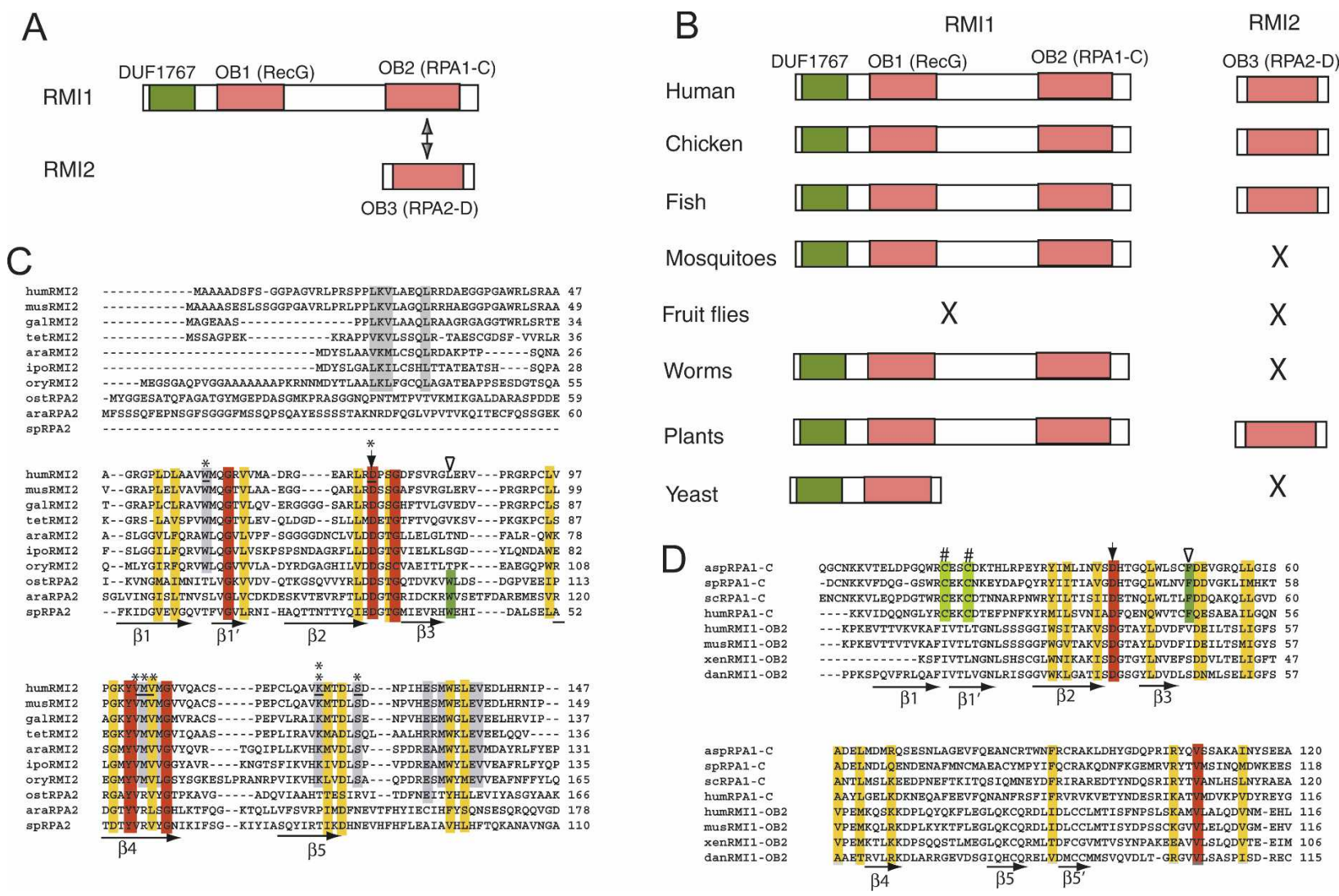

Figure 2. hRMI2 and hRMI1 contain three putative OB domains, two of which resemble a pair of interacting domains in RPA. (A) Schematic representation of hRMI1 and hRMI2. RMI1 contains two OB-fold domains: OB1 is most similar to the Wedge domain in bacterial RecG helicases (Supplemental Fig. 2), whereas OB2 resembles the RPA1-C domain (see D). RMI2 has one OB domain (OB3), which resembles that of RPA2-D (see $C$ ). DUF1767 is a domain of unknown function, but is conserved in RMI1 in all species. (B) Schematic representation of RMI1 and RMI2 in different eukaryotic species. The absence of an obvious ortholog is indicated by "X." The orthologs were identified by using the BLASTP algorithm to search the NR database maintained at NCBI. The orthologs include chicken RMI1 (NP_001026783) and RMI2 (NP_001006174.1); zebrafish RMI1 (AAH45482) and RMI2 (XP_691674.1); mosquito RMI1 (Anopheles gambiae XP_552585); worm RMI1 (C. elegans NP_741607); plant RMI1 (Arabidopsis thaliana NP_201159) and RMI2 (NP_172315.1); budding yeast RMI1 (Q02685). (C,D) Sequence alignment of RMI2 and RPA2 (C) or RMI1-OB2 and RPA1-C (D). The five predicted $\beta$-strands of the OB-folds are underlined with arrows. The identical and conserved residues are highlighted with red and yellow, respectively. Gray highlighted residues are only conserved in RMI2. Solid arrows indicate a highly conserved aspartic acid residue. Green highlighting and empty arrowheads mark the conserved aromatic residues conserved in RPA but not RMI. The residues mutated in Figure 7 are marked with an asterisk. K121, the mutation of which disrupts RMI2 function, is indicated by two asterisks. Two cysteine residues that are part of the Zn-finger in RPA1-C are marked with "\#," but these are not conserved in RMI1-OB2. The abbreviations and gene access numbers are as follows. RMI2: (mus) mouse, Q3UPE3; (gal) chicken; (tet) Tetraodon nigroviviridis, CAG1226.1; (ara) Arabidopsis thaliana, NP_172315.1; (ipo) Ipomoea trifida, BAF36320.1; (ory) Oryza sativa, AAK14410.1. RPA2: (ost) Ostreococcus tauri, CAL56444.1; (ara) Arabidopsis, NP_565571; (sp) Schizosaccharomyces pombe, NP_588227.2. RPA1: (asp) Aspergillus, XP_00127599.1; (sp) S. pombe, NP_595092.1; (sc) Saccharyomyces cerevisiae, NP_009404.1. RMI1: (xen) Xenopus 1aevis; (dan) Danio rerio. 
three subunits of the ssDNA-binding protein, RPA, which in total contains six OB-fold domains: RPA1 has four (RPA1-F, RPA1-A, RPA1-B, RPA1-C), whereas RPA2 and RPA3 have one each (RPA2-D and RPA3-E, respectively). RPA2-D contributes minimally to the DNA-binding activity of RPA, but is critical in mediating protein-protein interactions between RPA1-C and RPA3-E (Fanning et al. 2006). We searched the sequences of other BLM complex components for RPA-like OB-fold domains that could serve as interacting partners of RMI2-OB3. A previously defined OB domain in RMI (RMI1-OB1) (Yin et al. 2005) has no significant sequence similarity to the OB domains in RPA (Fig. 2A,2C). Instead, it is more similar to the "Wedge" domain in the bacterial helicase RecG (Supplemental Fig. 2), which has been suggested to bind and facilitate RecG-mediated unwinding of branched DNA (Briggs et al. 2005). Further analysis revealed that the C-terminal domain of RMI (termed RMI1-OB2) has sequence similarity to RPA1-C, the region required for interaction with RPA2-D (Fig. 2D). The similarity between the OB domains of RMI and RPA predict that RMI1-OB2 may interact with RMI2OB3 in a manner similar to that seen in their RPA counterparts (Fig. 2A).

In accord with this prediction, an RMI1 deletion mutant containing only OB2 coimmunoprecipitated with RMI2, but not with BLM or Topo $3 \alpha$ (Fig. 3A,B, lane 8). Moreover, an RMI1 mutant lacking OB2 coimmunoprecipitated with BLM and Topo $3 \alpha$, but not with RMI2 (Fig. $3 \mathrm{~B}$, lane 7). Thus, RMI1-OB2 is both necessary and sufficient for RMI1 to interact with RMI2. Moreover, these data demonstrate that the $\mathrm{N}$-terminal region of RMI1, which includes the conserved DUF1767 (Domain of Unknown function 1767, as named by Genebank) and OB1 domains, can form a stable complex with BLM and Topo $3 \alpha$ in the absence of RMI1-OB2 and RMI2. The results are consistent with findings that yeast RMI1 lacks the OB2 domain (Fig. 2C), but can form a complex with Sgs1 and Top3 (Chen and Brill 2007).

\section{Recombinant RMI1 and RMI2 form a stable complex}

The data above imply that the two RMI proteins likely form a complex through interactions between their RMI1-OB2 and RMI2-OB3 domains. We examined this possibility by coexpressing and purifying recombinant 6-histine-tagged RMI1 and strep-tagged RMI2 proteins in Escherichia coli (Supplemental Material). Recombinant RMI1 is highly insoluble when expressed in E. coli, and it cannot be analyzed without prior denaturation and renaturation (Wu et al. 2006). Coexpression with RMI2, however, rendered a significant fraction of RMI1 soluble (data not shown), suggesting that RMI2 promotes the proper folding of RMI1.

We purified the recombinant RMI1-RMI2 complex, "RMI," using a combination of conventional and affinity chromatography (see Supplemental Material). The elution profiles of the two proteins off the final gel filtration column were coincident, indicating that they are components of a single complex (Fig. 3C). The molecular mass of the complex was $\sim 180 \mathrm{kDa}$, consistent with it containing two molecules of each of RMI1 (75 kDa) and RMI2 (15 kDa).

The RMI complex promotes $d H J$ dissolution by $B L M$ and Topo $3 \alpha$

RMI1 can stimulate $\mathrm{dHJ}$ dissolution activity of BLM and Topo $3 \alpha$ (Raynard et al. 2006; Wu et al. 2006). We found that the recombinant RMI complex had a similar stimulatory activity in this assay (Fig. 3D; Supplemental Fig. 3A).

Attempts to directly compare the activity of the RMI complex versus RMIl itself were complicated by the fact that RMI1 is insoluble when expressed on its own in $E$. coli. To circumvent this problem, we expressed recombinant RMI1 in yeast (Supplemental Material), and found that it is soluble (Supplemental Fig. 4), and has a stimulatory activity comparable with that of the RMI complex on $\mathrm{dHJ}$ dissolution (Fig. 3D; Supplemental Fig. 3B). Moreover, we expressed and purified recombinant RMI2 in $E$. coli (Supplemental Fig. 5A) and found that it has no stimulatory effects on $\mathrm{dHJ}$ dissolution reactions containing BLM and Topo $3 \alpha$, either in the absence or the presence of RMI1 (Fig. 3D; Supplemental Fig. 3C; data not shown). These data suggest that the clear stimulatory activity of RMI complex on $\mathrm{dHJ}$ dissolution is mainly derived from RMI1, but not RMI2.

The RMI complex differs from other multi-OB-fold complexes in that it lacks detectable ssDNA-binding activity

All previously described multi-OB-fold complexes (RPA, TPP1-POT1, and Cdc13-Stn1-Ten1) can bind ssDNA. In our hands, $100 \mathrm{nM}$ recombinant RPA completely bound the ssDNA probe (Fig. 3E). In contrast, RMI failed to display detectable ssDNA-binding activity even at a 10fold higher concentration (Fig. 3E; see Supplemental Fig. 6 for ssDNA titration). Careful examination of the three OB domains of RMI revealed that they lack several critical residues that participate in DNA binding (Fig. 2C,D; Supplemental Fig. 2), which may explain the undetectable ssDNA-binding activity in RMI. Together, these data suggest that RMI may represent a new type of OBfold complex that acts through a mechanism other than by mediating protein-DNA interactions (see below).

\section{RMI2 is essential for the stability of BLM complexes}

Next we examined the contribution of RMI2 for BLM function in vivo. We found that HeLa cells depleted of RMI2 display reduced levels of BLM, Topo $3 \alpha$, and RMI1 (Fig. 4A,B), indicating that RMI2 is required for the overall stability of the BLM complex. Together with our previous findings that RMI1-depleted cells exhibit reduced levels of BLM and Topo $3 \alpha$ (Yin et al. 2005), the data suggest that the entire RMI complex is required for BLM complex stability. 

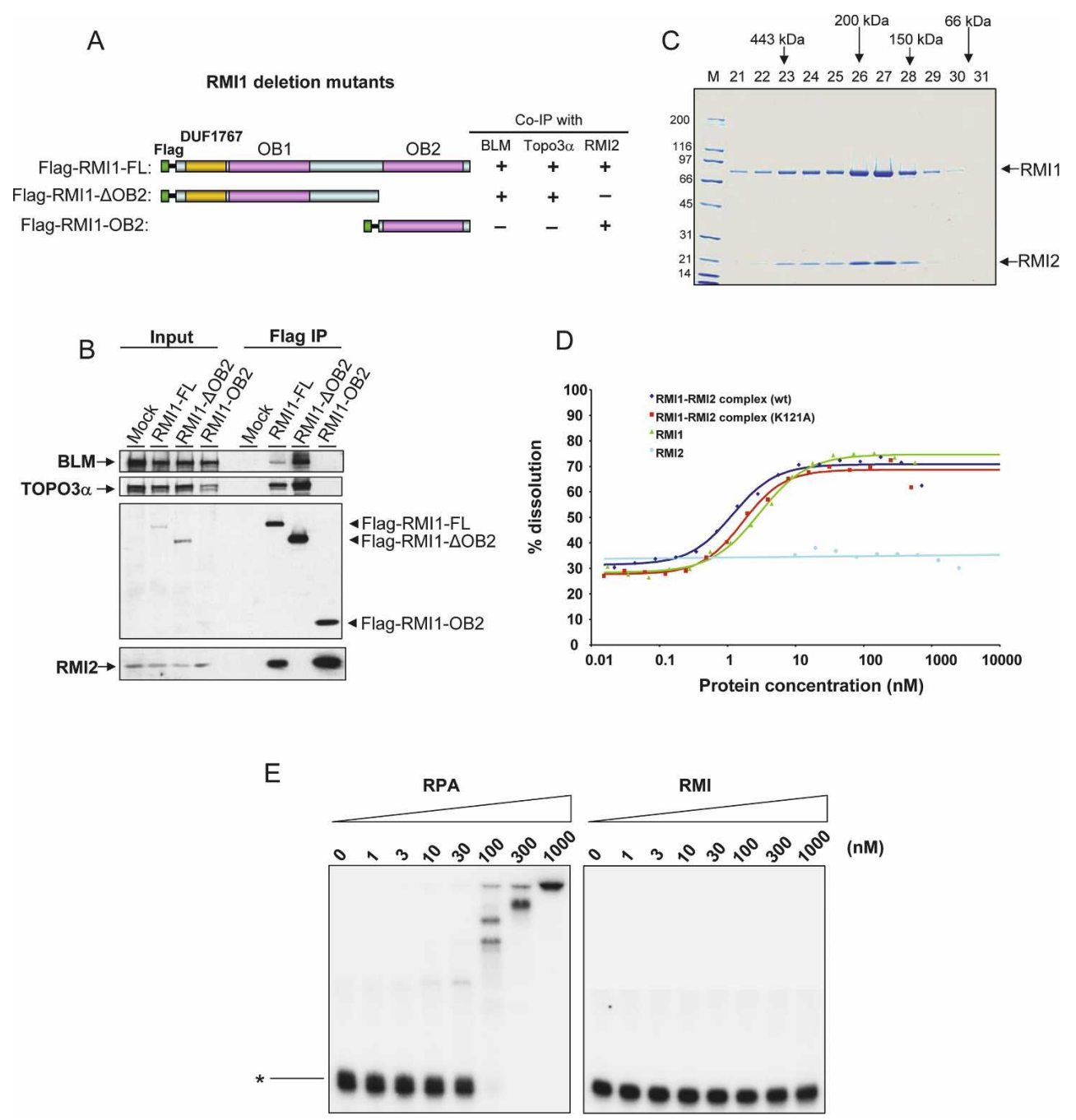

Figure 3. RMI2 and RMI1 constitute a new type of OB-fold complex that lacks detectable ssDNA-binding activity but is capable of stimulating $\mathrm{dHJ}$ dissolution. (A) Schematic representation of different RMIl deletion mutants (left), and their ability to coimmunoprecipitate with different BLM complex components from HeLa extract (right). (B) IP-Western showing that RMIl deletion mutants in $A$ coimmunoprecipitate with different BLM complex components. The Flag-tagged full-length (FL) or different deletion mutants of RMI1 were transfected into HeLa cells, and coimmunoprecipitation was performed using the Flag antibody. (C) A Coomassie-stained SDS-gel shows the purified recombinant RMI1-RMI2 complex fractionated by Sephadex 200 gel filtration column. RMI1 was fused with a 6xhistidine tag, whereas RMI2 was fused with a strep tag. The proteins were coexpressed in E. coli and were purified as described in Supplemental Material. Proteins with known molecular mass were used to calibrate the column, and their elution positions are shown above the figure. Notably, the peaks of the two RMI proteins are coincident, indicating that they are present in the same complex. (D) A graph showing stimulation of dHJ dissolution by the recombinant RMI complex containing wild-type RMI1-RMI2 (wt), RMI1-RMI2 (K121A) mutant, RMI1 purified from yeast, and RMI2. All reactions contained Topo $3 \alpha$ (22.5 nM) and BLM (10 nM). The primary image data of dissolution are shown in Supplemental Figure 3. (E) Gel-shift DNA-binding assay to show that the RMI complex exhibits little or no detectable ssDNA-binding activity (right panel) compared with RPA (left panel). Recombinant RPA complex was purified from E. coli and kindly provided by Dr. Guomin Li. A p32-labeled 94-nucleotide ssDNA is indicated on the left. The concentrations of different proteins are shown on the top.

Depletion of either RMI2, RMI1 or Topo $3 \alpha$, strongly decreased the levels of all three proteins $(60 \%-90 \%$; Fig. 4B), but only weakly reduced that of BLM $(20 \%-30 \%)$. Likewise, depletion of BLM drastically decreased its own level $(90 \%)$, but only modestly reduced the levels of the other three proteins $(10 \%-40 \%)$. These data are in accord with the findings that RMI1, RMI2, and Topo $3 \alpha$ can form a subcomplex without BLM (Supplemental Fig. 1). We also noticed that levels of RMI1 and RMI2 are highly dependent on one another, as depletion of either protein reduced the level of the other by $80 \%-90 \%$. This finding is also consistent with the data that RMI1 and RMI2 are direct interacting partners (Fig. 3).

\section{RMI2 is essential for mitotic phosphorylation of BLM}

BLM is known to be hyperphosphorylated in response to mitosis inhibitors Taxol and nocodazole, and this phos- 
Xu et al.

Figure 4. The RMI1-RMI2 complex is required for BLM complex stability and mitotic phosphorylation of BLM. $(A, B)$ Immunoblotting $(A)$, and its quantification $(B)$, to show that HeLa cells depleted of RMI2, RMI1, or Topo $3 \alpha$, exhibited reduced levels of other BLM complex components. Immunoblotting for $\beta$-actin was indent siRNA depletion experiments have been performed for each protein, and the immunoblotting data were quantified using TotalLab software. The protein level in cells treated with control siRNA was designated as 1 . Data represent the mean values, and the bars represent standard error. (C) Immunoblotting shows that mitotic phosphorylation of BLM is reduced in cells depleted of RMI2, RMI1, or Topo $3 \alpha$. Cells were either untreated (no drug), or treated with mitosis inhibitors Taxol $(1 \mu \mathrm{M})$ or Nocodazole $(100 \mathrm{ng} / \mathrm{mL})$ for $16 \mathrm{~h}$, as shown above the lanes. The hyperphosphorylated BLM is indicated (p-BLM). The ratio between hyper- and hypophosphorylated BLM was quantified using image software, and is shown below the image. cluded as a loading control. In $B$, at least four indepen-
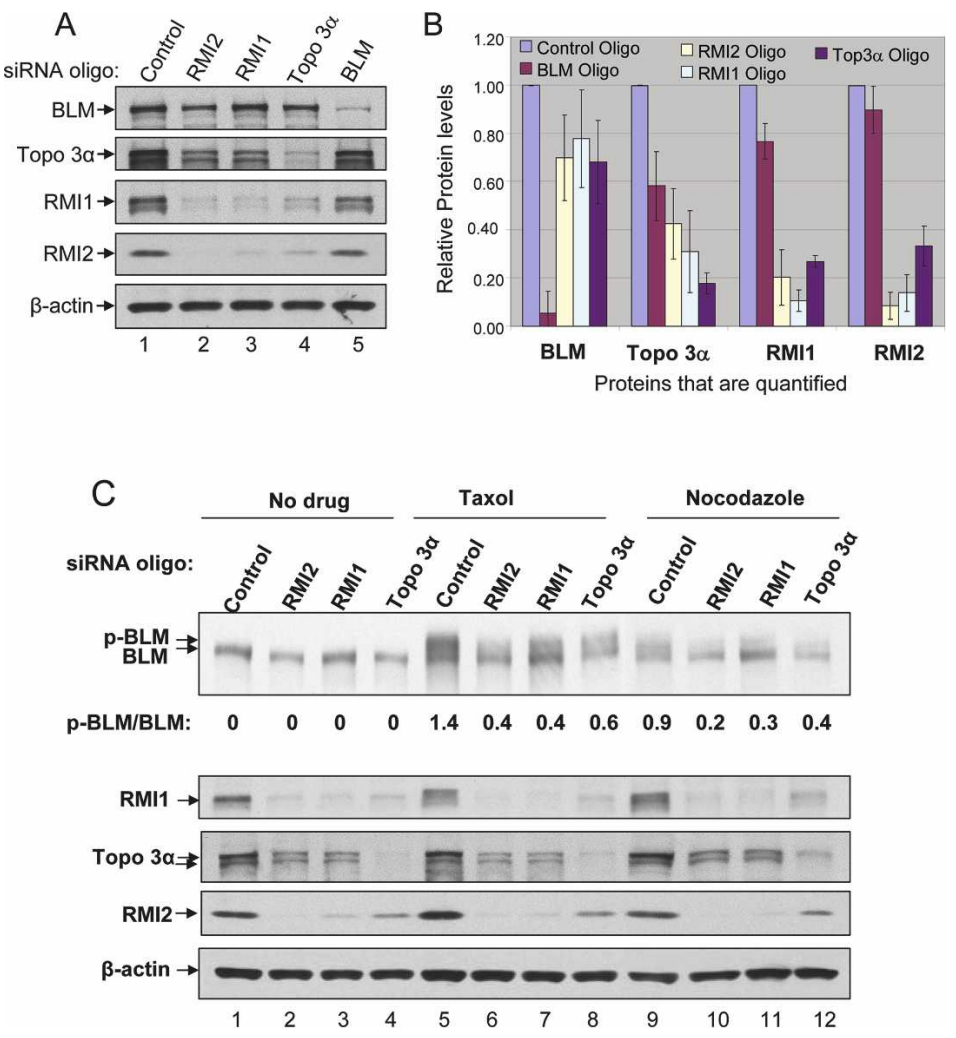

phorylation is required for normal chromosome segregation (Dutertre et al. 2000; Leng et al. 2006). The mitotic phosphorylation of BLM has been shown to be reduced in HeLa cells depleted of RMI1 (Yin et al. 2005). We found that BLM phosphorylation was similarly reduced in cells depleted of RMI2 (Fig. 4C, lanes 6,7,9,10). This result was expected, because cells depleted of RMI2 are concomitantly depleted of RMI1 (Fig. 4A). Moreover, BLM phosphorylation was also reduced in Topo $3 \alpha$-depleted cells (Fig. 5A). Thus, mitotic phosphorylation of BLM depends on the presence of its associated factors.

\section{RMI2 colocalizes with BLM and RMI1 in nuclear $D N A$ repair foci and is essential for focus formation}

BLM and RMIl have been shown to redistribute to discrete nuclear foci when cells are treated with DNA damaging agents or replication inhibitors (Y. Wang et al. 2000; Bischof et al. 2001; Wu et al. 2001; Yin et al. 2005). Proteins in these foci, such as RAD51 and other DNA repair factors, play important roles in DNA repair (Wu et al. 2001; Davalos et al. 2004). We found that Flag-tagged RMI2 could also redistribute to nuclear foci in response to DNA damaging agents and replication inhibitors, including mitomycin $\mathrm{C}$ (MMC), hydroxyurea, and aphidicolin (Fig. 5A). Moreover, RMI2-containing foci colocalize with those of RMI1 and BLM (Fig. 5B,C), providing additional in vivo evidence that RMI2 is part of the BLM complex (Fig. 1).

We showed previously that the number of nuclear foci containing BLM is reduced in RMI1-depleted cells (Yin et al. 2005). We performed similar analyses in RMI2depleted HeLa cells (see Fig. 4 for the efficiency of depletion), and found that the number of BLM foci was again reduced (Fig. 5D), indicating that the entire RMI complex is essential for BLM recruitment or retention in nuclear foci containing RMI1 is also reduced in RMI2depleted cells (Fig. 5D), which supports the notion that RMI2 is required for correct localization of the entire BLM complex to the sites of DNA damage.

\section{RMI2 suppresses SCE via the same pathway as BLM}

The hallmark feature of BS cells is elevated SCE levels, typically about 10 -fold higher than those seen in normal cells (Chaganti et al. 1974; German 1993). A twofold increase in SCE levels has been observed previously in BLM complex is involved in suppressing SCE (Yin et al. 2005). To examine if RMI2 is also required for the same process, we disrupted the homologous gene in chicken DT40 cells. This cell line has a very high gene-targeting efficiency, and has been widely used for functional analysis of the genes involved in DNA repair pathways, including BLM and Topo $3 \alpha$ (W. Wang et al. 2000; Seki et al. 2006).

We generated two targeting vectors for the chicken RMI2 gene (Fig. 6A) and used them sequentially to inactivate both of its alleles, as confirmed by Southern blotting, genomic-PCR, and RT-PCR analysis (Fig. 6B-D). Immunoblotting revealed that the levels of RMI1 and DNA damage sites. We observed that the number of HeLa cells depleted of RMIl, suggesting that the entire 

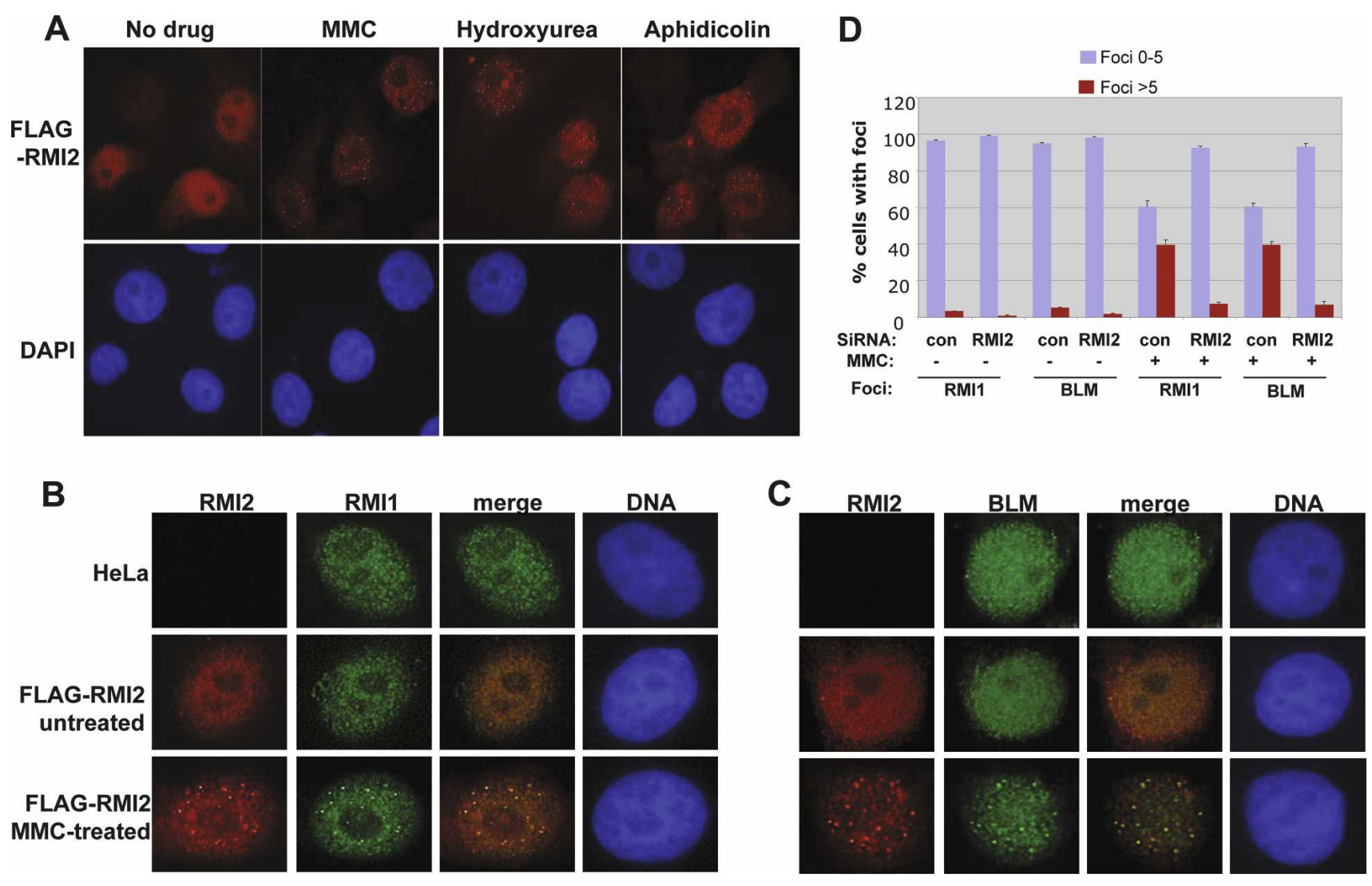

Figure 5. RMI2 colocalizes with BLM and RMI1 in nuclear foci in response to DNA damage, and is required for focus formation. (A) Immunofluorescence showing that RMI2 is redistributed to nuclear foci in response to several DNA damaging agents, including MMC, hydroxyurea, and aphidicolin. $(B, C)$ Immunofluorescence showing that Flag-tagged RMI2 (Flag-RMI2) colocalizes with RMI1 (B) and $\operatorname{BLM}(C)$ in nuclear foci in response to MMC treatment. HeLa cells that are not transfected with Flag-RMI2 were included as a negative control. $(D)$ A histogram to illustrate that the percentage of cells containing BLM or RMIl foci is drastically reduced in HeLa cells depleted of RMI2 in response to MMC treatment.

Topo $3 \alpha$ were reduced in $R M I 2^{-/-}$cells (Fig. $\left.6 \mathrm{E}\right)$. This is consistent with the siRNA data showing that RMI2 is required for stability of these two proteins (Fig. 4). Unfortunately, our antibodies against human RMI2 (hRMI2) and BLM did not recognize their chicken homologs, precluding us from detecting them by immunoblotting.

The $R M I 2^{-/-}$cells obtained displayed an SCE frequency about five- to sixfold higher than that of wild-type cells (11 vs. 2) (Fig. 6F), indicating that RMI2 is required to suppress SCE. The SCE frequency of $R M I 2^{-/-}$cells was lower than that of $B L M^{-/-}$cells (11 vs. 19), hinting that the BLM complex is partially active without RMI2. We performed epistasis analyses by inactivating $R M I 2$ in $B L M^{-/-}$cells (data not shown). The $R M I 2^{-/-} / B L M^{-/-}$double-knockout cells displayed an SCE level comparable with that of $B L M^{-/-}$cells (18 vs. 19) (Fig. 6F), indicating that RMI2 suppresses SCE through the same pathway as does BLM.

$R M I 2^{-/-}$cells lack certain phenotypes characteristic of $B L M^{-/-}$or Topo $3 \alpha^{-/-}$cells, indicating that RMI2 is not required for all of the functions of the BLM complex. Specifically, $R M I 2^{-/-}$cells lacked the cellular hypersensitivity to cisplatin and MMS (Supplemental Fig. 7A,B) observed in $B L M^{-/-}$cells (W. Wang et al. 2000; Hirano et al. 2005), and $R M I 2^{-/-} / B L M^{-/-}$double-knockout cells exhibited the same degree of hypersensitivity as that of $B L M^{-/-}$cells (Supplemental Fig. 7A,B). These data suggest that RMI2 is dispensable for BLM to repair or bypass DNA damage induced by these drugs in DT40 cells.

$R M I 2^{-/-}$cells also displayed a proliferation rate comparable with that of wild-type cells (Supplemental Fig. $7 \mathrm{C})$, in contrast to Topo $3 \alpha^{-/-}$cells that are nonviable (Seki et al. 2006), indicating that RMI2 is dispensable for Topo $3 \alpha$ to mediate an essential function in cell proliferation. Interestingly, $R M I 2^{-/-} / B L M^{-/-}$cells exhibited a growth rate slower than $B L M^{-/-}$cells, suggesting that RMI2 plays a role in cell proliferation in the absence of BLM. This role of RMI2 might be related to its requirement for the stability of Topo $3 \alpha$; because Topo $3 \alpha$ is essential for cell proliferation (Seki et al. 2006), its reduced stability in the absence of RMI2 may lead to reduced growth rate in the $R M I 2^{-/-} / B L M^{-/-}$cells.

The interaction between RMI2 and BLM is essential for the suppression of SCE

We stably expressed hRMI2 in RMI2-/- DT40 cells, and found that hRMI2 restored the protein levels of the 
Xu et al.
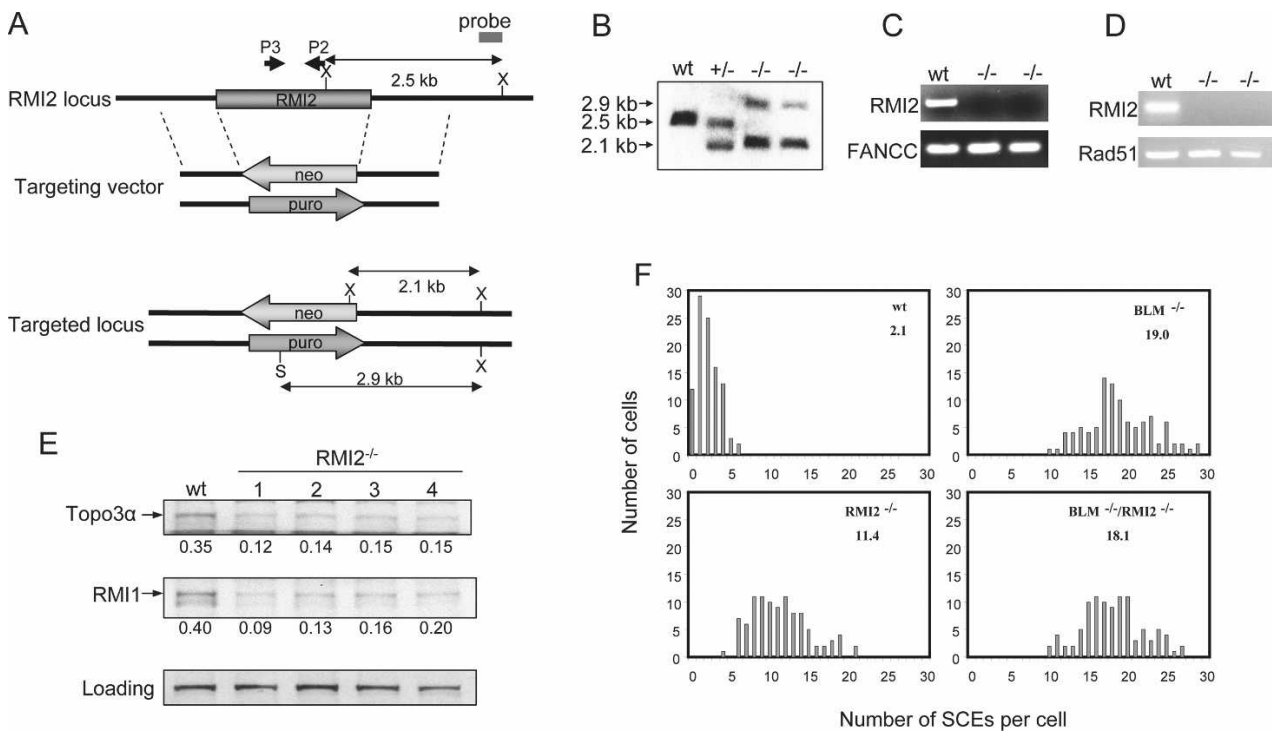

Figure 6. RMI2-null DT40 cells resemble BLM mutant cells in displaying an elevated level of SCE. (A) Schematic representation of the chicken RMI2 genomic DNA, the two targeting vectors, and the restriction map of the locus after knockout. Two restriction enzyme digestion sites used in Southern Blotting, XhoI and SalI, are marked as X and S, respectively. The primers used for genomicPCR analysis (P3-P2) are depicted in B. Southern blotting shows that both alleles of the RMI2 gene have been inactivated by the targeting vectors described in $A$. The genomic DNA from wild-type $(\mathrm{wt}), R M I 2^{+/-}$, and $R M I 2^{-/-}$cells was first digested with XhoI and SalI restriction enzymes, and subsequently hybridized with the probe shown in $A$. (C) Genomic-PCR analysis to show the absence of RMI2 genomic DNA in $R M I 2^{-/-}$cells. The FANCC gene was included as a positive control. (D) RT-PCR analysis to show that RMI2 mRNA is undetectable in $R M I 2^{-/-}$cells. RAD51 was included as a positive control. (E) Immunoblotting to show that Topo $3 \alpha$ and RMI1 levels are reduced in four different clones of $R M I 2^{-/-}$cells. $(F)$ Histograms showing the SCE levels of wild-type, $R M I 2^{-/-}$cells, $\mathrm{BLM}^{-/-}$, and $\mathrm{RMI}^{-/-} / \mathrm{BLM}^{-/-}$cells. The mean number of SCEs per cell is shown in the top right corner.

chicken RMI1 and Topo $3 \alpha$ proteins to those of wild-type cells (Fig. 7A,B). This is consistent with the data above that RMI2 is required for the stability of BLM complex components (Figs. 4A, 6E). Moreover, hRMI2 reduced the elevated SCE level to that of wild-type cells (Fig. 7A,C), further supporting a proposed important role of RMI2 in the suppression of SCE.

The finding that hRMI2 can functionally substitute for its chicken homolog allowed us to use this system for structure-function analyses of RMI2. We substituted several conserved residues within the RMI2-OB3 domain with alanine (Fig. 7A; see Fig. 2B for sequence alignment), and stably expressed the mutant proteins in $R_{M I 2^{-I-}}$ cells (Fig. $7 \mathrm{~B}$ ). All of the mutant proteins were expressed at comparable levels and could restore the stability of Topo $3 \alpha$ and RMI1 (Fig. 7A,B). Moreover, all of them coimmunoprecipited with Topo $3 \alpha$ and RMIl with efficiency similar to that of the wild-type protein (Fig. 7B).

Interestingly, the K121A mutant (MutE) and the K121A/S126A double-mutant (MutD) failed to complement the abnormal SCE phenotype, whereas the S126A mutant could complement, indicating that $\mathrm{K} 121$ is critical for RMI2 to suppress SCE (Fig. 7A,C). Because K121A mutant can restore the reduced levels of Topo $3 \alpha$ and RMI1 in $R M I 2^{-/-}$cells to those of wild-type cells, the instability of the BLM complex is unlikely to be the cause of the higher SCE frequency in $R M I 2^{-/-}$DT40 cells.
To assess the defect in the K121A mutant, we stably expressed HF-tagged RMI2-K121A protein in HeLa cells. Immunoblotting showed that this mutant coimmunoprecipitated with a drastically reduced level of BLM, but near normal amounts of Topo $3 \alpha$ and RMI1 (Fig. 7D). These data indicate that the K121A substitution disrupts protein-protein interactions between BLM and the Topo 3 $\alpha-$ RMI1RMI2 complex that is critical for suppression of SCE.

Next, we purified from E. coli a recombinant complex containing RMI1 and the RMI2-K121A point mutant (Supplemental Fig. 5B). This mutant complex was indistinguishable from the wild-type complex in stimulating dHJ dissolution (Fig. 3D; Supplemental Fig. 3D), suggesting that the interaction between RMI2 and BLM is dispensable for $\mathrm{dHJ}$ dissolution, at least in vitro.

\section{Discussion}

RMI2 is a new component of the BLM complex essential for the maintenance of genome stability

Biochemical and genetic studies have demonstrated that RMIl is an integral component of the BLM complex essential for the maintenance of genome stability. Here, we identified RMI2 as a new component of the BLM complex using the same biochemical approach as was used for identification of RMI1 (Fig. 1). Importantly, genetic analyses in chicken DT40 cells demonstrated that vertebrate cells lacking RMI2 have a higher level of SCE, 
GENESDEV/2008/105015, Figure 7, Xu et al.

A

\begin{tabular}{|c|c|c|c|c|c|c|}
\hline \multirow{3}{*}{ 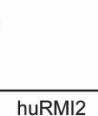 } & \multirow{2}{*}{ Proteins } & \multirow{2}{*}{$\begin{array}{l}\text { Complementation } \\
\text { of SCE }\end{array}$} & \multirow{2}{*}{$\begin{array}{l}\text { Complementation } \\
\text { of stability of } \\
\text { RMI1 and Topo } 3 \alpha\end{array}$} & \multicolumn{3}{|c|}{ Co-IP with } \\
\hline & & & & RMI1 & Topo3 $\alpha$ & BLM \\
\hline & 1 & + & + & + & + & + \\
\hline MutA & W59A & + & + & + & + & N.D. \\
\hline MutB & $\begin{array}{l}076 A \\
\end{array}$ & + & + & + & + & N.D. \\
\hline MutC & V102AM103A V104A & + & + & + & + & N.D. \\
\hline MutD & K121A $\$ 126 \mathrm{~A}$ & 4 & + & + & + & N.D. \\
\hline MutE & K121A & - & + & + & + & - \\
\hline MutF & $\begin{array}{c}\text { OB-Fold } \\
\text { OB }\end{array}$ & + & + & + & + & N.D. \\
\hline
\end{tabular}
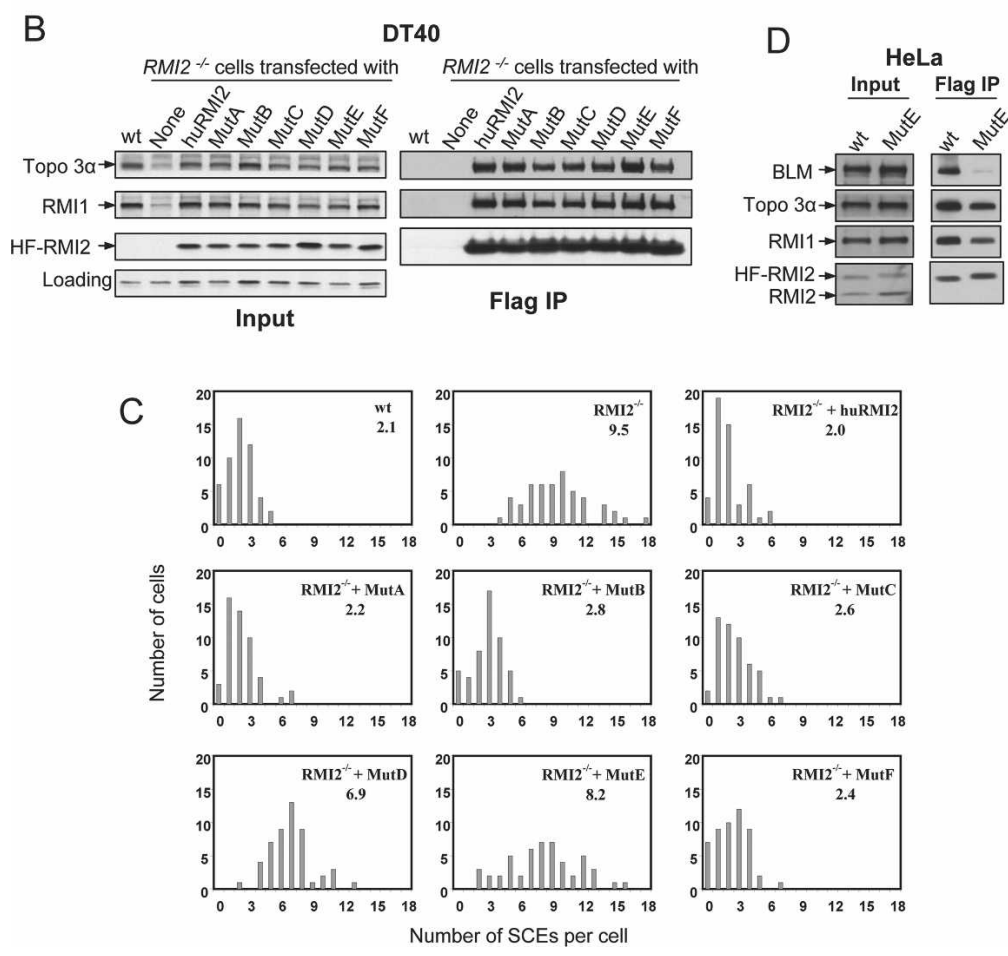

Figure 7. An RMI2 point mutant defective in mediating interactions with BLM is deficient in suppressing SCE in RMI2 mutant cells. (A) Schematic representation of different hRMI2 point mutants and their ability to suppress the elevated SCE level $(C)$ and restore the protein stability of RMI1 and Topo $3 \alpha(B)$ in $R M I 2^{-/-}$DT40 cells. Their ability to interact with BLM complex components based on $B$ and $D$ are also shown. The amino acid residues mutated are shown in Figure 2C. (N.D) Not determined. $(B$, left panels) Immunoblotting showing that human wild-type and different point mutants of RMI2 can complement the phenotype of reduced stability of Topo $3 \alpha$ and RMIl of RMI $2^{-/-}$cells. (Right panels) Moreover, these point mutants can efficiently coimmunoprecipitate with Topo $3 \alpha$ and RMI1, indicating that their ability to interact with these two proteins remains largely intact. The wild-type and mutant RMI2 are all fused to a Flag-epitope, and immunoprecipitation was performed using the Flag antibody. (C) Histograms showing SCE levels of $R M I 2^{-/-}$DT40 cells complemented by wild-type and various point mutants of hRMI2 as illustrated in $A$. The mean number of SCEs per cell is shown in the top right corner. (D) Immunoblotting shows that the K121A mutant (MutE) coimmunoprecipitated with drastically reduced level of BLM, indicating that this mutant is defective in interacting with BLM. the hallmark feature of BS cells (Fig. 6), and reintroduction of RMI2 into $R M I 2^{-/-}$cells suppressed the SCE level to that of wild-type cells (Fig. 7). Moreover, $R M I 2^{-/-} / B L M^{-/-}$ double-knockout cells exhibited a level of SCE similar to that of $B L M^{-/-}$cells, indicating that RMI2 and BLM suppress SCE through the same pathway. These data demonstrate that, like RMI1, RMI2 is a component of the BLM complex and is essential for BLM to maintain genome stability.

Previous epistasis analyses have shown that the SCE increase in $B L M^{-/-}$DT40 cells is largely due to increased HR events (W. Wang et al. 2000), which is in agreement with the current model that BLM plays an important role in HR (Wu and Hickson 2006). Because RMI2 suppresses SCE through the same pathway as does BLM (Fig. 6F), the elevated SCE frequency in $R M I 2^{-/-}$cells is most likely also due to increased HR events. Our data therefore favor a model that RMI2 plays a role in HR by mediating BLM function.

One consequence of increased HR is that it can result in increased loss of heterozygosity, leading to inactiva- tion of tumor suppressor genes and development of cancer. The phenotypic similarity between $R M I 2^{-/-}$and $B L M^{--}$cells in SCE (HR) increase, and the physical and functional connection between the two proteins predict that mutations in RMI2 may be responsible for disease conditions similar to those seen in BS, including cancer predisposition. Indeed, carriers of a genetic variant of RMI1 have been suggested to exhibit an increased cancer risk (Broberg et al. 2007). By analogy, mutations or genetic variants in RMI2 gene may also lead to genomic instability and increased cancer risk. Inactivating this gene in mice may help to test this hypothesis.

$R_{M I 2^{-/-}}$DT40 cells lack certain phenotypes observed in $B L M^{-/-}$cells, including cellular hypersensitivity to DNA damage agents (Supplemental Fig. 7) and chromosomal breakage (data not shown). Unlike the SCE increase that is a hallmark feature of all BLM-deficient cells, these two phenotypes are associated with many other genomic instability diseases, such as Fanconi anemia and ataxia telangiectasia. Moreover, the DNA damage 
sensitivity is absent in certain BLM-mutant cells (Honma et al. 2002; Marple et al. 2006). Thus, RMI2 seems to be specifically important for the hallmark function of BLM in maintaining genome stability-the suppression of SCE-but dispensable for other BLM functions, at least in DT40 cells. In this regard, the laboratories of Drs. Meetei and Sung (Singh et al. 2008) have independently identified RMI2 in the BLM complex, and demonstrated that human HEK293 cells depleted of RMI2 display MMS hypersensitivity and increased chromosomal breakage. Therefore, the requirement of RMI2 for BLM function in genome maintenance may vary depending on the specific cell types.

\section{RMI2 and RMI1 constitute an essential structural and functional unit in the BLM complex}

Several lines of evidence suggest that RMI1 requires RMI2 for its stability and function, and that they work together as a heteromeric unit. First, RMI1 is highly unstable in vertebrate cells that lack or have reduced levels of RMI2 (Figs. 4, 6). Second, recombinant RMI1 is essentially insoluble in E. coli without coexpression of RMI2. Third, recombinant RMI1 and RMI2 form the highly stable RMI complex that is resistant to dissociation using $2 \mathrm{M} \mathrm{NaCl}$ (data not shown), and is capable of stimulating dHJ dissolution (Fig. 3; Supplemental Fig. 3A). Fourth, both RMI complex components are required for the stability, mitotic phosphorylation, and nuclear focus localization of the BLM complex (Figs. 4, 5). Finally, both proteins are required for BLM to suppress SCE, as evidenced by elevated SCE levels in RMI1-depleted HeLa cells (Yin et al. 2005), and in RMI2-inactivated DT40 cells (Fig. 6).

RMI1 has been suggested to play two critical roles in the BLM complex: First, it serves as a structural component to allow proper assembly and or stability of the complex (Yin et al. 2005); second, it stimulates the binding and dissolution of $\mathrm{dHJ}$ (Raynard et al. 2006; Wu et al. 2006) or single HJ (Bussen et al. 2007; Chen and Brill 2007). Our findings that both RMI1 and RMI2 are required for BLM complex stability, and that the RMI complex is capable of stimulating $\mathrm{dHJ}$ dissolution, suggest that the entire RMI complex plays these two roles in the BLM complex.

\section{The OB domains of RMI mainly act by mediating important protein-protein interactions, rather than protein-DNA interactions}

A prominent feature of the RMI complex is the presence of three putative $\mathrm{OB}$ domains, which resemble other multi-OB-fold complexes involved in DNA repair and protection of telomeres, such as RPA, TPP1-POT1, and Cdc13-Stn1-Ten1. OB domains commonly function by binding to ssDNA, an intermediate frequently produced during DNA processing events (Theobald et al. 2003). Indeed, both human and yeast RMI1 have been shown to bind ssDNA and HJ DNA in vitro (Mullen et al. 2005; Wu et al. 2006; Chen and Brill 2007; Raynard et al. 2008). However, this binding is of very low affinity, as the dis- sociation constant $(\mathrm{Kd})$ for the binding reaction is $>100$ $\mathrm{nM}$, higher than that of Topo $3 \alpha(\mathrm{Kd} \sim 10 \mathrm{nM})$ (Chen and Brill 2007) and RPA (between 0.1 and $1 \mathrm{nM}$ ) (Fanning et al. 2006). Moreover, a hRMI1 mutant deleted of its DNAbinding domain (which encompasses OB2) is proficient in stimulating $\mathrm{dHJ}$ dissolution in vitro (Raynard et al. 2008), which argues against a major role of DNA binding in RMI1 function.

Our findings that the OB domains of RMI lack several conserved residues that are important for DNA binding in RPA or RecG imply that RMI may have weak or no DNA-binding activity. Specifically, although RMI1-OB2 has sequence similarity to RPA1-C, it lacks the conserved Zinc-finger of the latter, which has been shown to be critical for DNA binding (Bochkareva et al. 2000). Consistent with this notion, we failed to detect any significant DNA-binding activity for the RMI complex at up to $1000 \mathrm{nM}$ protein concentration, using ssDNA (Fig. 3E), dsDNA, Y-shaped DNA, forked DNA, or HJ DNA as substrates (data not shown). However, we cannot rule out the possibilities that RMI may have transient or weak DNA-binding activity that cannot be detected by the current assay, or that RMI may bind DNA only in a complex with Topo $3 \alpha$ and BLM.

Our data favor the hypothesis that OB domains of RMI function mainly by mediating critical protein-protein interactions. In agreement with this model, two OB domains in the RMI complex, RMI1-OB2 and RMI2-OB3, have sequence similarity to a pair of interacting OB domains in RPA (Fig. 2). Our coimmunoprecipitation experiments showed that RMI1-OB2 is both necessary and sufficient to interact with RMI2 (Fig. 4), supporting the notion that OB2 and OB3 participate in specific proteinprotein interactions like similar domains in their RPA counterparts. Further support for this model comes from the finding that the $\mathrm{K} 121 \mathrm{~A}$ point substitution in the RMI2-OB3 domain disrupts the interaction between BLM and the Topo $3 \alpha$-RMI subcomplex, and abrogates the ability of RMI2 to suppress the elevated SCE level of RMI2 $^{-/-}$DT40 cells (Fig. 7). Moreover, Sung and colleagues (Raynard et al. 2008) have shown that a point mutant in RMI1-OB1 abolishes the interaction between RMI1 and Topo $3 \alpha$, and inactivates its ability to stimulate $\mathrm{dHJ}$ dissolution. Speculatively, the interactions among RMI, BLM, and Topo $3 \alpha$ may facilitate important conformational changes that increase the stability and activity of the latter two proteins.

It is somewhat puzzling that our K121A mutant fails to suppress SCE (Fig. 7) but is proficient in stimulating $\mathrm{dHJ}$ dissolution (Fig. 3D). Possibly, the interactions among proteins or between proteins and DNA may be tightly controlled in the highly organized nucleus, but such control may be lost in the test tube, where proteins and DNA can freely diffuse and interact.

\section{Two multi-OB-fold complexes mediate BLM function}

In conclusion, we discovered a novel multi-OB-fold complex that differs from other similar complexes in that its 
major function appears to mediate protein-protein, rather than protein-DNA, interactions. As such, the BLM complex contains two multi-OB-fold complexes that can promote its catalytic activities: RPA and RMI (Plank et al. 2006; Raynard et al. 2006; Wu et al. 2006; this study). RPA can bind ssDNA to promote the DNA unwinding activity of BLM (Brosh et al. 2000), whereas RMI interacts with Topo $3 \alpha$ and BLM, and stimulates $\mathrm{dHJ}$ dissolution. Although protein-protein interactions appear to be dispensable for $\mathrm{dHJ}$ dissolution in vitro (Wu et al. 2006), they are required for full suppression of SCE in vivo (Hu et al. 2001). The interaction of RMI with BLM and Topo $3 \mathrm{a}$ enhances not only the activities of the latter two proteins, but also potentially creates a bridge that allows coordinated action of the complex during the catalysis of $\mathrm{dHJ}$ dissolution in vivo. Moreover, it is possible that the concerted action of both RPA and RMI may be needed in some cases for efficient dissolution of $\mathrm{dHJ}$ and genome maintenance by BLM.

\section{Materials and methods}

\section{Cell culture and transfection}

HeLa cells were cultured in DMEM supplemented with $10 \%$ fetal calf serum. To establish stable cell line expressing HFRMI2 (6-His and Flag-tagged), HeLa cells were transfected with plasmid pIRES-puro3-FlagRMI2 using Lipofectamine (Invitrogen) and selected in growth medium containing puromycin (1 $\mu \mathrm{g} / \mathrm{mL}$ ). For transient transfection of HF-RMI2, HeLa cells were transfected as described above but in the absence of puromycin. For DNA damage induction, HeLa cells were treated with MMC $(50 \mathrm{ng} / \mathrm{mL})$, hydroxyurea $(1 \mathrm{mM})$, or aphidicolin $(4 \mu \mathrm{M})$ for $18 \mathrm{~h}$.

Transfections of siRNAs were carried out with Oligofectamine (Invitrogen). siRNA oligos for BLM (AGCAGCGAUG UGAUUUGCA) and RMI1 (AGCCUUCACGAAUGUUGAU) were synthesized by Dharmacon. The smart pool oligos of RMI2, Topo $3 \alpha$, and control oligos were also from Dharmacon. The control oligos are either ON-TARGETplus siCONTROL Non-Targeting Pool or single oligo (AUUGUAUGCGAUCGCA GAC).

DT40 cells were cultured at $39.5^{\circ} \mathrm{C}$ in RPMI 1640 medium supplemented with $10 \%$ fetal calf serum, $1 \%$ chicken serum, 10 mM HEPES, and penicillin-streptomycin mixture. Transfection was performed using electroporation with an Amaxa Nucleofector2 and Solution T. Cells were selected using medium containing G418 $(2 \mathrm{mg} / \mathrm{mL})$ or puromycin $(0.5 \mu \mathrm{g} / \mathrm{mL})$.

\section{dHJ dissolution assay}

The $\mathrm{dHJ}$ dissolution assay was performed as described (Wu et al. 2006).

\section{DNA-binding assay}

Recombinant RMI or RPA were incubated at $37^{\circ} \mathrm{C}$ with $1.25 \mathrm{nM}$ p32-labeled ssDNA substrate (TCCTTTTGATAAGAGGTCAT TTTT GCGGAT GGCTT AGAGCTTAATT GCTGAATCTGG TGCTGTAGGTCAACATGTT GTAAATATGCAGCT AAAG) in $10 \mu \mathrm{L}$ of reaction buffer $(25 \mathrm{mM}$ Tris- $\mathrm{HCl}$ at $\mathrm{pH} 7.5,2 \mathrm{mM}$ $\mathrm{MgCl}_{2}, 60 \mathrm{mM} \mathrm{KCl}, 1 \mathrm{mM} \mathrm{DTT}, 100 \mu \mathrm{g} / \mathrm{mL} \mathrm{BSA}$ ) for $15 \mathrm{~min}$. Reaction mixture was loaded and resolved on a $6 \%$ DNA Retardation Gel (Invitrogen).

\section{Gel filtration analysis}

Nuclear extracts were prepared according to the protocol described previously (Yin et al. 2005). The nuclear pellet was only extracted once with buffer $\mathrm{C}$ (20 mM HEPES at pH 7.9, $0.42 \mathrm{M}$ $\mathrm{NaCl}, 25 \%$ glycerol, $1.5 \mathrm{mM} \mathrm{MgCl}{ }_{2}, 0.2 \mathrm{mM}$ EDTA). The extract was then loaded onto a Superose 6 column (HR16/50; Amersham Pharmacia Biotechnology), which had already been equilibrated with buffer (20 mM HEPES at pH 7.9, $200 \mathrm{mM}$ $\mathrm{NaCl}, 1 \mathrm{mM}$ dithiothreitol [DTT], $0.1 \mathrm{mM}$ phenylmethylsulfonyl fluoride [PMSF], 0.1\% Tween 20, 5\% glycerol). Fractions were analyzed by SDS-PAGE and immunoblotting.

\section{Immunoprecipitation}

Immunoprecipitation was performed using the protocol described previously (Yin et al. 2005). A rabbit RMI2 polyclonal antibody was generated against a fusion protein containing fulllength RMI2 fused to the maltose-binding protein (New England Biolabs). Polyclonal antibodies against BLM, Topo $3 \alpha$ or RMI1 were described elsewhere (Wu et al. 2000; Meetei et al. 2003; Yin et al. 2005). $\beta$-actin and RPA32 antibodies were from Bethyl Laboratories. RPA70 antibody was from Calbiochem.

In experiments described in Figure 3A, cells were lysed in NP buffer (25 mM Tris- $\mathrm{HCl}$ at $\mathrm{pH} 7.5,150 \mathrm{mM} \mathrm{NaCl}, 0.5 \% \mathrm{NP}-40$, $5 \%$ glycerol, $1 \mathrm{mM}$ DTT, protease inhibitor cocktail, $1 \mathrm{mM}$ PMSF). Coimmunoprecipitation was performed using the antiFlag M2-agarose (Sigma).

\section{Generation of the DT40 knockout strains}

To generate RMI2-knockout vectors, two RMI2 genomic DNA fragments were amplified by PCR using two pairs of primers: TATTTGACATGACAGAAGTGAGTG/AGGGATCCTGTTT TCTGCTCTCTGTAAC and AGGGATCCCCTGAATACTTC TGCCTGAAAG/AGGG TACCTGTAACTCC TCT ATGTATG. The two DNA fragments were used as the $5^{\prime}$ and $3^{\prime}$ arms in the knockout constructs, respectively. They were first cloned separately into the pCR2.1-TOPO vector (InVitrogen). Then, the 3' arm was subcloned into the BamHI/KpnI sites of pCR2.1-TOPO vector containing the $5^{\prime} \mathrm{arm}$. The neomycin or puromycin drugresistant gene cassettes were subsequently cloned into the BamHI site.

For Southern blotting, a 0.3-kb RMI2 probe was generated by PCR amplification of genomic DNA using primers ACTCCTC TAGCCCTGCCTTGCAGAG/CTGTACAAGTTCACAGCTG TTTC. For RT-PCR analysis of the RMI2 mRNA levels, two primers P1 (CAGCGGCCACTTCACCGTGCT) and P2 (GTT TTCGGAAAGATCCGTCATC) were used. For genomic-PCR analysis to confirm the deletion of the RMI2 genomic DNA, primers P3 (CTTTCCCTCTCCTCCCGCTCAT) and P2 were used.

The $B L M^{-/-}$DT40 cells have been described previously (Otsuki et al. 2007). BLM ${ }^{-/-} / R_{M I 2^{-/-}}$double-knockout cells were generated by deleting RMI2 gene in $B L M^{-/-}$cells using the same vectors.

For complementation of $R M I 2^{-/-}$DT40 cells, HF-tagged hRMI2 was inserted to the pCDNA3.1-Zeo expression vector (Invitrogen). HF-RMI2 point mutants were generated by using QuikChange mutagenesis kit (Stratagene). The plasmids expressing HF-RMI2 wild-type or mutant proteins were transfected into $\mathrm{RMI}^{-/-}$cells. Those that stably express the proteins were selected by immunoblotting.

Additional experimental procedures are described in Supplemental Material. 


\section{Acknowledgments}

We thank Drs. A.R. Meetei for communicating unpublished data, G. Li for recombinant RPA, and D. Schlessinger for critical reading of the manuscript. This work was supported in part by the Intramural Research Program of the National Institute on Aging (Z01 AG000657-08), National Institutes of Health; and by the Canadian Institutes of Health Research (MOP-79368).

\section{References}

Bachrati, C.Z. and Hickson, I.D. 2008. RecQ helicases: Guardian angels of the DNA replication fork. Chromosoma 117: 219-233.

Bachrati, C.Z., Borts, R.H., and Hickson, I.D. 2006. Mobile Dloops are a preferred substrate for the Bloom's syndrome helicase. Nucleic Acids Res. 34: 2269-2279.

Bischof, O., Kim, S.H., Irving, J., Beresten, S., Ellis, N.A., and Campisi, J. 2001. Regulation and localization of the Bloom syndrome protein in response to DNA damage. J. Cell Biol. 153: $367-380$.

Bochkareva, E., Korolev, S., and Bochkarev, A. 2000. The role for zinc in replication protein A. J. Biol. Chem. 275: 2733227338.

Briggs, G.S., Mahdi, A.A., Wen, Q., and Lloyd, R.G. 2005. DNA binding by the substrate specificity (wedge) domain of RecG helicase suggests a role in processivity. J. Biol. Chem. 280: 13921-13927.

Broberg, K., Hoglund, M., Gustafsson, C., Bjork, J., Ingvar, C., Albin, M., and Olsson, H. 2007. Genetic variant of the human homologous recombination-associated gene RMI (S455N) impacts the risk of AML/MDS and malignant melanoma. Cancer Lett. 258: 38-44.

Brosh Jr., R.M., Li, J.L., Kenny, M.K., Karow, J.K., Cooper, M.P., Kureekattil, R.P., Hickson, I.D., and Bohr, V.A. 2000. Replication protein A physically interacts with the Bloom's syndrome protein and stimulates its helicase activity. J. Biol. Chem. 275: 23500-23508.

Bugreev, D.V., Yu, X., Egelman, E.H., and Mazin, A.V. 2007. Novel pro- and anti-recombination activities of the Bloom's syndrome helicase. Genes \& Dev. 21: 3085-3094.

Bussen, W., Raynard, S., Busygina, V., Singh, A.K., and Sung, P. 2007. Holliday junction processing activity of the BLM-Topo III $\alpha$-BLAP75 complex. J. Biol. Chem. 282: 31484-31492.

Chaganti, R.S., Schonberg, S., and German, J. 1974. A manyfold increase in sister chromatid exchanges in Bloom's syndrome lymphocytes. Proc. Natl. Acad. Sci. 71: 4508-4512.

Chang, M., Bellaoui, M., Zhang, C., Desai, R., Morozov, P., Delgado-Cruzata, L., Rothstein, R., Freyer, G.A., Boone, C., and Brown, G.W. 2005. RMI1/NCE4, a suppressor of genome instability, encodes a member of the RecQ helicase/Topo III complex. EMBO J. 24: 2024-2033.

Chen, C.F. and Brill, S.J. 2007. Binding and activation of DNA topoisomerase III by the Rmil subunit. J. Biol. Chem. 282: 28971-28979.

Davalos, A.R., Kaminker, P., Hansen, R.K., and Campisi, J. 2004. ATR and ATM-dependent movement of BLM helicase during replication stress ensures optimal ATM activation and 53BP1 focus formation. Cell Cycle 3: 1579-1586.

Dutertre, S., Ababou, M., Onclercq, R., Delic, J., Chatton, B., Jaulin, C., and Amor-Gueret, M. 2000. Cell cycle regulation of the endogenous wild type Bloom's syndrome DNA helicase. Oncogene 19: 2731-2738.

Fanning, E., Klimovich, V., and Nager, A.R. 2006. A dynamic model for replication protein A (RPA) function in DNA processing pathways. Nucleic Acids Res. 34: 4126-4137.
German, J. 1993. Bloom syndrome: A mendelian prototype of somatic mutational disease. Medicine 72: 393-406.

Hirano, S., Yamamoto, K., Ishiai, M., Yamazoe, M., Seki, M., Matsushita, N., Ohzeki, M., Yamashita, Y.M., Arakawa, H., Buerstedde, J.M., et al. 2005. Functional relationships of FANCC to homologous recombination, translesion synthesis, and BLM. EMBO J. 24: 418-427.

Honma, M., Tadokoro, S., Sakamoto, H., Tanabe, H., Sugimoto, M., Furuichi, Y., Satoh, T., Sofuni, T., Goto, M., and Hayashi, M. 2002. Chromosomal instability in B-lymphoblasotoid cell lines from Werner and Bloom syndrome patients. Mutat. Res. 520: 15-24.

Hu, P., Beresten, S.F., van Brabant, A.J., Ye, T.Z., Pandolfi, P.P., Johnson, F.B., Guarente, L., and Ellis, N.A. 2001. Evidence for BLM and topoisomerase III $\alpha$ interaction in genomic stability. Hum. Mol. Genet. 10: 1287-1298.

Karow, J.K., Constantinou, A., Li, J.L., West, S.C., and Hickson, I.D. 2000. The Bloom's syndrome gene product promotes branch migration of holliday junctions. Proc. Natl. Acad. Sci. 97: 6504-6508.

Leng, M., Chan, D.W., Luo, H., Zhu, C., Qin, J., and Wang, Y. 2006. MPS1-dependent mitotic BLM phosphorylation is important for chromosome stability. Proc. Natl. Acad. Sci. 103: $11485-11490$.

Marple, T., Kim, T.M., and Hasty, P. 2006. Embryonic stem cells deficient for Brca2 or Blm exhibit divergent genotoxic profiles that support opposing activities during homologous recombination. Mutat. Res. 602: 110-120.

Meetei, A.R., Sechi, S., Wallisch, M., Yang, D., Young, M.K., Joenje, H., Hoatlin, M.E., and Wang, W. 2003. A multiprotein nuclear complex connects Fanconi anemia and Bloom syndrome. Mol. Cell. Biol. 23: 3417-3426.

Meetei, A.R., Medhurst, A.L., Ling, C., Xue, Y., Singh, T.R., Bier, P., Steltenpool, J., Stone, S., Dokal, I., Mathew, C.G., et al. 2005. A human ortholog of archaeal DNA repair protein Hef is defective in Fanconi anemia complementation group M. Nat. Genet. 37: 958-963.

Mullen, J.R., Nallaseth, F.S., Lan, Y.Q., Slagle, C.E., and Brill, S.J. 2005. Yeast Rmil/Nce4 controls genome stability as a subunit of the Sgs1-Top3 complex. Mol. Cell. Biol. 25: 44764487.

Otsuki, M., Seki, M., Inoue, E., Yoshimura, A., Kato, G., Yamanouchi, S., Kawabe, Y., Tada, S., Shinohara, A., Komura, J., et al. 2007. Functional interactions between BLM and XRCC3 in the cell. J. Cell Biol. 179: 53-63.

Plank, J.L., Wu, J., and Hsieh, T.S. 2006. Topoisomerase III $\alpha$ and Bloom's helicase can resolve a mobile double Holliday junction substrate through convergent branch migration. Proc. Natl. Acad. Sci. 103: 11118-11123.

Ralf, C., Hickson, I.D., and Wu, L. 2006. The Bloom's syndrome helicase can promote the regression of a model replication fork. J. Biol. Chem. 281: 22839-22846.

Raynard, S., Bussen, W., and Sung, P. 2006. A double Holliday junction dissolvasome comprising BLM, topoisomerase III $\alpha$, and BLAP75. I. Biol. Chem. 281: 13861-13864.

Raynard, S., Zhao, W., Bussen, W., Lu, L., Ding, Y.Y., Busygina, V., Meetei, A.R., and Sung, P. 2008. Functional role of BLAP75 in BLM/Topo III $\alpha$-dependent holliday junction processing. J. Biol. Chem. 283: 15701-15708.

Singh, T.R., Ali, A.M., Busygina, V., Raynard, S., Fan, Q., Du, C.-h., Andreasson, P.R., Sung, P. and Meetei, A.R. 2008 BLAP18/RMI2, a novel OB-fold-containing protein, is an essential component of the Bloom helicase-double Holliday junction dissolvasome. Genes \& Dev. (this issue). doi: 10.1101/ gad.1725108.

Seki, M., Nakagawa, T., Seki, T., Kato, G., Tada, S., Takahashi, 
Y., Yoshimura, A., Kobayashi, T., Aoki, A., Otsuki, M., et al. 2006. Bloom helicase and DNA topoisomerase III $\alpha$ are involved in the dissolution of sister chromatids. Mol. Cell. Biol. 26: 6299-6307.

Theobald, D.L., Mitton-Fry, R.M., and Wuttke, D.S. 2003. Nucleic acid recognition by OB-fold proteins. Annu. Rev. Biophys. Biomol. Struct. 32: 115-133.

van Brabant, A.J., Ye, T., Sanz, M., German, I.J., Ellis, N.A., and Holloman, W.K. 2000. Binding and melting of D-loops by the Bloom syndrome helicase. Biochemistry 39: 14617-14625.

Wang, W., Seki, M., Narita, Y., Sonoda, E., Takeda, S., Yamada, K., Masuko, T., Katada, T., and Enomoto, T. 2000. Possible association of BLM in decreasing DNA double strand breaks during DNA replication. EMBO J. 19: 3428-3435.

Wang, Y., Cortez, D., Yazdi, P., Neff, N., Elledge, S.J., and Qin, J. 2000. BASC, a super complex of BRCA1-associated proteins involved in the recognition and repair of aberrant DNA structures. Genes \& Dev. 14: 927-939.

Wu, L. and Hickson, I.D. 2003. The Bloom's syndrome helicase suppresses crossing over during homologous recombination. Nature 426: 870-874.

Wu, L. and Hickson, I.D. 2006. DNA helicases required for homologous recombination and repair of damaged replication forks. Annu. Rev. Genet. 40: 279-306.

Wu, L., Davies, S.L., North, P.S., Goulaouic, H., Riou, J.F., Turley, H., Gatter, K.C., and Hickson, I.D. 2000. The Bloom's syndrome gene product interacts with topoisomerase III. $J$. Biol. Chem. 275: 9636-9644.

Wu, L., Davies, S.L., Levitt, N.C., and Hickson, I.D. 2001. Potential role for the BLM helicase in recombinational repair via a conserved interaction with RAD51. J. Biol. Chem. 276: 19375-19381.

Wu, L., Bachrati, C.Z., Ou, J., Xu, C., Yin, J., Chang, M., Wang, W., Li, L., Brown, G.W., and Hickson, I.D. 2006. BLAP75/ RMI1 promotes the BLM-dependent dissolution of homologous recombination intermediates. Proc. Natl. Acad. Sci. 103: 4068-4073.

Yin, J., Sobeck, A., Xu, C., Meetei, A.R., Hoatlin, M., Li, L., and Wang, W. 2005. BLAP75, an essential component of Bloom's syndrome protein complexes that maintain genome integrity. $E M B O$ I. 24: 1465-1476. 


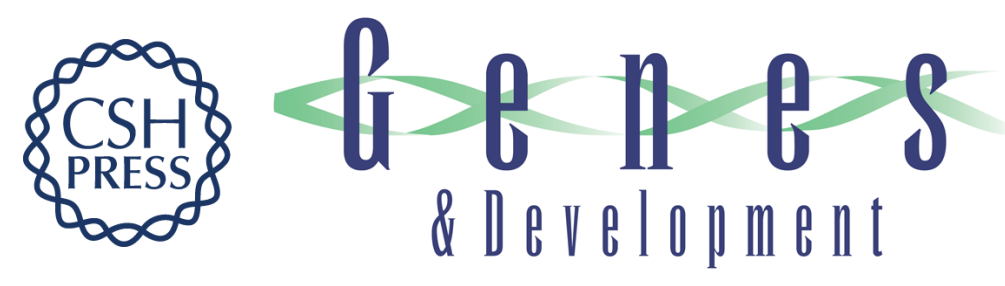

\section{RMI, a new OB-fold complex essential for Bloom syndrome protein to maintain genome stability}

Dongyi Xu, Rong Guo, Alexandra Sobeck, et al.

Genes Dev. 2008, 22:

Access the most recent version at doi:10.1101/gad.1708608

\section{Supplemental http://genesdev.cshlp.org/content/suppl/2008/10/17/22.20.2843.DC1 Material}

Related Content

References

\section{License}

Email Alerting Service
This article cites 41 articles, 24 of which can be accessed free at: http://genesdev.cshlp.org/content/22/20/2843.full.html\#ref-list-1

Articles cited in:

http://genesdev.cshlp.org/content/22/20/2843.full.html\#related-urls

More complexity to the Blooms syndrome complex

Yilun Liu and Stephen C. West

Genes Dev. October , 2008 22: 2737-2742

Receive free email alerts when new articles cite this article - sign up in the box at the top right corner of the article or click here.

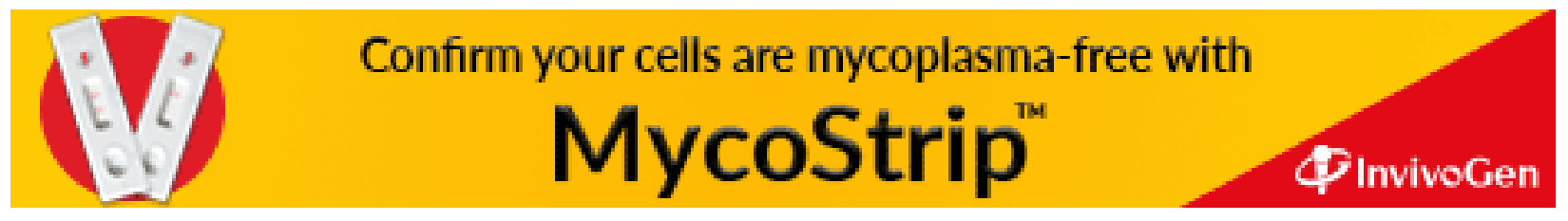

